\title{
Health system readiness to support facilities for care of preterm, low birth weight, and sick newborns in Ethiopia: a qualitative assessment
}

Abubeker Kedir Usman ${ }^{1}$, Eskinder Wolka², Yared Tadesse ${ }^{3}$, Abraham Tariku $^{3}$, Abate Yeshidinber ${ }^{4}$, Alula M. Teklu4, Kirsten Senturia ${ }^{5}$, Wendemaghen Gezahegn ${ }^{4}$, James A. Litch ${ }^{5^{*}}$ (i) and And the Every Preemie-SCALE Ethiopia Implementation Research Collaboration Group

\begin{abstract}
Background: Preterm birth is a worldwide challenge with the highest burden in low- and middle-income countries. Despite availability of low-cost interventions to decrease mortality of preterm, low birth weight, and sick newborns, these interventions are not well integrated in the health systems of low- and middle-income countries. The aim of this study was to assess, from the perspective of key stakeholders comprising leaders in the public health system, the health system readiness to support health care facilities in the care provided to preterm, low birth weight, and sick newborns in different regions of Ethiopia.

Methods: A qualitative assessment using in-depth interviews with health facility leaders was conducted in health facilities in 3 regions of Ethiopia from December 2017 to February 2018. The interview guide was developed using a modified version of the World Health Organization health system building blocks.
\end{abstract}

Results: Across the public health system, adequate and reliable space, power, and water were problematic. Human resource issues (training, staffing, and retention) were critical to being able to properly care for preterm, low birth weight, and sick newborns. Problems with functional equipment and equipment distribution systems were widespread. Funds were lacking to support preterm, low birth weight, and sick newborn needs in facilities. Data collection practices, data quality, and data utilization were all problematic. There were gaps in the availability of guidelines and protocols, specifically targeting preterm, low birth weight, and sick newborn care. Key facilitators, information disseminators, and influencers identified in the study were the Health Development Army, community and religious leaders, and mothers and families who had had positive experiences or outcomes of care.

Conclusions: The Ethiopian health system has opportunities across all 7 World Health Organization health system building blocks to strengthen readiness to support health facilities to provide quality care and improve outcomes for preterm, low birth weight, and sick newborns.

Keywords: Health system, Health system readiness, Preterm, Low birth weight, Sick newborn, Newborn, Newborn health, Qualitative research, Ethiopia

\footnotetext{
* Correspondence: jim.litch@gapps.org; jlitch@yahoo.com

${ }^{5}$ Global Alliance to Prevent Prematurity and Stillbirth (GAPPS), 19009 33rd

Avenue W, Suite 200, Lynnwood, WA 98036, USA

Full list of author information is available at the end of the article
}

(c) The Author(s). 2019 Open Access This article is distributed under the terms of the Creative Commons Attribution 4.0 International License (http://creativecommons.org/licenses/by/4.0/), which permits unrestricted use, distribution, and reproduction in any medium, provided you give appropriate credit to the original author(s) and the source, provide a link to the Creative Commons license, and indicate if changes were made. The Creative Commons Public Domain Dedication waiver (http://creativecommons.org/publicdomain/zero/1.0/) applies to the data made available in this article, unless otherwise stated. 


\section{Background}

It is estimated that 15 million (11.1\%) babies born worldwide in 2010 were preterm (born alive before 37 weeks gestation) [1]. Preterm birth is a worldwide challenge, with the highest burden in low- and middle-income countries (LMICs). Despite the availability of low-cost interventions to reduce mortality of preterm, low birth weight (LBW), and sick newborns, these interventions are not well integrated in the health systems of LMICs. Neonatal deaths are the leading cause of under-5 mortality [2], and complications of prematurity is the leading cause of neonatal mortality [3, 4].

Through substantial investments in public-sector health systems, Ethiopia has achieved notable progress over the last decade in preventing postnatal newborn deaths. Newborns benefit from high-impact interventions that include integrated community case management, community-based newborn care, and prevention of mother-to-child transmission of HIV through Option $\mathrm{B}+$. However, reductions in neonatal mortality have lagged behind reductions in child mortality [4]. The country has one of the highest neonatal mortality rates globally, with 28 neonatal deaths per 1000 live births, and has the sixth highest number of neonatal deaths, with an estimated 90,000 deaths in 2016 [5]. Of under-5 deaths in Ethiopia, 44\% occur within the first 28 days of life, a rate that has remained stagnant. The most common causes of death among newborns in Ethiopia are prematurity (37\%), infection (28\%), and asphyxia or other intrapartum events (24\%) [6].

Countries with high neonatal mortality have bottlenecks in almost all of the health system building blocks defined by the World Health Organization (WHO): service delivery, health workforce, health management information systems, access to essential medicines, financing, leadership and governance, and community engagement $[7,8]$. A study from 12 high-burden countries showed that the most prevalent bottlenecks were in the areas of health workforce and health financing (10 out of 12 countries), followed by community ownership and partnership (9 out of 12 countries) [9].

Establishment of neonatal intensive care units (NICUs) and diversified levels of care have increased the survival of preterm, LBW, and sick newborns less than 32 weeks of gestation, although this may not apply to most countries with the highest burden of preterm deliveries and deaths [10]. Despite the absence of these high-level centers, moderately preterm newborns can be saved with feasible, cost-effective care [11]. Barriers to neonatal care include shortages of qualified personnel, geographical inaccessibility, inadequate regulation of pharmaceutical and private sector providers, insufficient provider knowledge and skills, poor demand for interventions, and lack of equipment and infrastructure. Continuous and multifaceted improvement in the established health system improves the overall outcome of neonates $[12,13]$.

The aim of this study was to assess, from the perspective of key stakeholders comprising leaders in the public health system, the Government of Ethiopia health system's readiness to support health care facilities in the care provided to preterm, LBW, and sick newborns in 3 different regions of Ethiopia. For the purposes of this paper, we define health system readiness as the preparedness of the system, including all components-facilities, staff, and organizational structure - to provide appropriate evidence based care for preterm, LBW, and sick newborns primarily from 28 weeks gestational age and above.

\section{Methods}

\section{Study design}

A team of researchers from Ethiopia and the United States conducted a qualitative assessment in multiple sites across Ethiopia to describe facility readiness to deliver care services to preterm, LBW, and sick newborns, by assessing service availability and key processes.

From December 2017 to February 2018, data collectors conducted key informant interviews with facility leaders in the public health care system, many of whom were also clinical care providers delivering obstetric and newborn care services in study health facilities. Qualitative methods were used to gather rich data to inform how and why the health system needs improvements in serving preterm, LBW, and sick newborns, rather than to establish prevalence numbers. Taking a narrative approach, we used thematic content analysis to answer specific a priori questions about the Ethiopian health system. This report conforms to the Standards for Reporting Qualitative Research [14].

The WHO health systems building blocks provide a framework for understanding and assessing the key components of a health system. Within each block, key domains and the critical factors that influence structure, function, and outcome of that domain are described. This study used a modified version of the health systems building block framework to guide our assessment of the current context of services for preterm, LBW, and sick newborns in Ethiopia [15].

\section{Study sites}

The Federal Democratic Republic of Ethiopia is among the most populated countries in Africa, with a population estimate of 107 million for 2018 [16]. One urban and 2 rural regions were selected to allow for assessment and description of different settings and contexts in Ethiopia representing an urban community, a settled agrarian community, and a semi-pastoral agrarian community.

Staff and clients from a subset of health facilities at each tier of the public health care system in each region 
participated, including specialized hospitals, general hospitals, primary hospitals, health centers, and health posts [17].

\section{Data tools}

A new tool was developed for this project using a multistep process. First, based on the WHO framework, the scope of the tool was defined, and questions were drafted. After their initial training, data collectors pretested the instrument at St. Paul's Hospital Millennium Medical College with 5 interviewees similar to the target sample. Adjustments were made for flow, content, terms used, prompts, and instructions.

Using this structured interview guide, facility leaders were asked questions about policies and guidelines, programs, facility preparedness, and referral transfer systems, all with respect to preterm, LBW, and sick newborns (see Additional file 1).

\section{Sampling}

We used purposive convenience sampling to recruit from all clinical, nursing, and administrative leaders who were involved in the oversight of units or care programs for pregnancy, labor, and delivery/obstetrics, and gynecological, postnatal, and newborn care services at the study health facilities. We interviewed 37 leaders from all public specialized, general, and primary hospitals and a subset of health centers and health posts located within the 3 study regions (see Table 2).

\section{Saturation}

Interview data were reviewed from all 3 study regions periodically during data collection until data saturation [18] was reached, according to the judgment of the core team.

\section{Research team composition, training, and supervision}

The research team was composed of core members, data collectors, and data analysts. Experienced data collectors were trained on the study overview, objectives, participant selection, instruments, and interview skills. Supervision was conducted throughout data collection in all 3 study regions using a supervision checklist.

\section{Data collection and management \\ Recruitment}

Trained study interviewers identified facility administrators who identified department leaders eligible to participate in the study. Interviewers invited and scheduled potential participants for in-person interviews.

\section{Location}

Each interview was conducted in the leader's office at a time convenient for the interviewee. Participants were offered their choice of languages (Amharic or Oromiffa) for the interview.

\section{Recording, transcribing, and translation}

Demographic data were recorded on a tablet and interviews were recorded digitally. Recordings were transcribed into the local written language by experienced transcriptionists and were subsequently translated by professional translators. Transcriptions and translations were spot-checked for accuracy by a third team member. Every effort was made to maintain participants' confidentiality both during data collection and during report writing and publication. All audiotapes were destroyed immediately following transcription. No names are attached to any of the data. In the results, quotes are identified only by location $(\mathrm{AMH}=\mathrm{Amhara}, \mathrm{ORO}=$ Oromia, $\mathrm{AA}=$ Addis Ababa) where relevant, and by facility level $(\mathrm{HP}=$ health post, $\mathrm{HC}=$ health center, $\mathrm{HOSP}=$ hospital $)$ where relevant to the findings.

\section{Analysis}

Data were entered into NVivo version 12 and analyzed using thematic content analysis [19]. Researchers involved in the study design and qualitative coders developed a codebook and applied it to the coding of all transcripts.

All transcripts were open-coded using the final version of the codebook to capture key themes and relevant ideas as identified in the data. Each transcript was coded by 2 separate coders, and disagreements were resolved by the lead analyst who reviewed all discrepancies and discussed them with the second coder as necessary to reconcile coding. Once coding was complete, code reports were produced for each code, cleaned, and prepared for synthesis.

Each code report was synthesized by one team member as follows: initially, text excerpts no longer appearing relevant to the code were grayed out; remaining excerpts were annotated with comments; particularly illustrative quotes were highlighted; and comments were summarized into a table of theme domains and subdomains with associated quotes for each code report.

\section{Results}

\section{Description of sample}

All respondents were from Addis Ababa, Amhara Region, and Oromia Region. The characteristics of study regions are shown in Table 1.

Clinical leaders were proportionally divided across all 3 regions; $50 \%$ were 26 to 30 years of age; and all were employed as midwives, nurses, health officers, heath extension workers (HEWs), or neonatologists (Table 2). 
Table 1 Characteristics of study regions ${ }^{a}$

\begin{tabular}{|c|c|c|c|c|}
\hline Characteristics & National & Addis Ababa & Amhara & Oromia \\
\hline \multicolumn{5}{|l|}{ Demographic indicators } \\
\hline Population, No. ${ }^{\mathrm{b}}$ & $73,918,505$ & $2,738,248$ & $17,214,056$ & $27,158,471$ \\
\hline Proportion urban population, $\%{ }^{\mathrm{b}}$ & 16.2 & 100.0 & 12.3 & 12.4 \\
\hline Total fertility rate, No. of children per woman & 4.6 & 1.8 & 3.7 & 5.4 \\
\hline Proportion of women who are literate, $\%$ & 42.0 & 87.8 & 44.9 & 37.3 \\
\hline Proportion of women who own/use a bank account, \% & 15.1 & 53.6 & 20.9 & 8.4 \\
\hline Proportion of women who own/use a mobile phone, $\%$ & 27.3 & 87.0 & 21.2 & 23.3 \\
\hline Proportion of men who are engaged in agriculture, $\%$ & 71 & 1 & 62 & 41 \\
\hline \multicolumn{5}{|l|}{ Mortality rates } \\
\hline Under-5 mortality, No. per 1000 live births & 67 & 39 & 85 & 79 \\
\hline Infant mortality, No. per 1000 live births & 48 & 28 & 67 & 60 \\
\hline Neonatal mortality, No. per 1000 live births & 29 & 18 & 47 & 37 \\
\hline Low birth weight rate, \% & 12.7 & 11.5 & 22.2 & 13.1 \\
\hline \multicolumn{5}{|l|}{ Maternal and child health services indicators } \\
\hline Proportion of pregnant women who received antenatal care from a skilled provider, \% & 28.0 & 96.8 & 67.1 & 50.7 \\
\hline Proportion of deliveries in a health facility, $\%$ & 26.0 & 96.6 & 27.1 & 18.8 \\
\hline Proportion of women with a postnatal checkup in first $2 \mathrm{~d}$ after birth, \% & 17.0 & 55.4 & 21.9 & 11.8 \\
\hline Proportion of children (ages 12-23 mo) who received all basic vaccinations by $12 \mathrm{mo} \%$ & 22.0 & 81.6 & 39.9 & 24.3 \\
\hline
\end{tabular}

a Data from the Ethiopia Demographic and Health Survey 2016, except as denoted in footnote b [20]

${ }^{\mathrm{b}}$ Data from the 2007 National Census [21]

\section{Health service delivery \\ Infrastructure}

Across the public health system, adequate and reliable space, power, and water functionality were problematic. Table 3 displays the issues linked by facility levels.

\section{Service readiness}

Participants were asked how ready their local health system was to deliver (1) routine care to newborns and (2) care to preterm, LBW, and sick newborns-across labor and delivery, NICU, kangaroo mother care (KMC), and inpatient wards. Only hospitals and advanced health centers have specialized care units, but all leaders were asked about readiness for newborn care and preterm, LBW, and sick newborn care. A few leaders reported being very equipped to provide care, but most cited problems in one or more areas that inhibited effective provision of care. Table 4 shows the challenges related to readiness in each of the facility units.

\section{Health workforce and human resources}

Human resource issues (training, staffing, and retention) were critical to being able to properly care for preterm, LBW, and sick newborns.

\section{Provider training and skills}

Clinical leaders saw staff training as mandatory for quality service provision and specifically recommended training in newborn care, including refresher training that did not currently exist: "There should be constant providers and the number of providers need to be increased; one provider is needed for one baby if they are critically sick; if possible one nurse should be assigned for one baby. To do so, the number of health providers matters" (AA-HOSP). Training on newborn care basics was not common for staff in general. Staff preservice training in the preterm, LBW, and sick newborn program was inadequate for many, if not most, providers. Labor and delivery training was limited and training applicable to preterm, LBW, and sick newborn care was primarily related to breastfeeding and KMC. Previously, nongovernmental organizations had provided some training in care for preterm, LBW, and sick newborns, but that was no longer happening.

Lack of training was identified as one factor leading to unnecessary referrals. Inadequate numbers of trained providers-especially senior staff, skilled professionals, and NICU staff-were also seen as limitations to system readiness. The difficulty of getting a newborn with a complication to an appropriate specialist led to loss of life. Furthermore, high turnover of trained professionals was an ongoing problem.

\section{Communication}

Functionality also depended upon effective communication between providers in the same facility and in other facilities. Participants reported that meetings were the 
Table 2 Background characteristics of facility leaders

\begin{tabular}{|c|c|c|}
\hline Characteristics & Leaders, No. $(n=37)$ & Percentage \\
\hline \multicolumn{3}{|l|}{$\overline{\text { Age, } y^{a}}$} \\
\hline 20 to 25 & 6 & 16.7 \\
\hline 26 to 30 & 18 & 50.0 \\
\hline 31 to 35 & 6 & 16.7 \\
\hline 36 to 40 & 4 & 11.1 \\
\hline 41 to 45 & 2 & 5.6 \\
\hline \multicolumn{3}{|l|}{ Sex } \\
\hline Female & 21 & 56.8 \\
\hline Male & 16 & 43.2 \\
\hline \multicolumn{3}{|l|}{ Profession } \\
\hline Midwife & 1 & 2.7 \\
\hline Nurse & 15 & 40.5 \\
\hline Health officer & 7 & 18.9 \\
\hline Health extension worker & 13 & 35.1 \\
\hline Neonatologist & 1 & 2.7 \\
\hline \multicolumn{3}{|l|}{ Position } \\
\hline Head nurse & 1 & 2.7 \\
\hline Head, health center & 5 & 13.5 \\
\hline Head, health post & 15 & 40.5 \\
\hline Head, maternal child health & 1 & 2.7 \\
\hline Head, NICU & 3 & 8.1 \\
\hline Head, pediatric department & 1 & 2.7 \\
\hline Lead, labor and delivery & 1 & 2.7 \\
\hline Medical director & 9 & 24.3 \\
\hline Coordinator, NICU & 1 & 2.7 \\
\hline \multicolumn{3}{|l|}{ Facility level } \\
\hline Specialized hospital & 2 & 5.4 \\
\hline General hospital & 3 & 8.1 \\
\hline Primary hospital & 1 & 2.7 \\
\hline Health center & 16 & 43.2 \\
\hline Health post & 15 & 40.5 \\
\hline \multicolumn{3}{|l|}{ Region } \\
\hline Addis Ababa & 12 & 32.4 \\
\hline Amhara & 14 & 37.8 \\
\hline Oromia & 11 & 29.7 \\
\hline
\end{tabular}

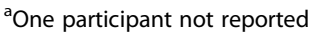

Abbreviation: NICU neonatal intensive care unit

primary form of communication. Using catchment area meetings at hospitals to bring health center and hospital staff together to communicate or consult on cases seemed widespread. Meetings were also regularly held within facilities to communicate about specific cases or deliver training updates, in the style of grand rounds seminars. Health centers communicated with health posts via midwives and with HEWs via conference meetings. This may have been limited to certain facilities and not universal. Changes to guidelines, policies, and trainings were conveyed through written communication from clinical leaders.

Participants recognized the importance of communication for effective care of preterm, LBW, and sick newborns and called for improvements both within units at facilities and between levels of facilities: "To improve these challenges, I think we need to communicate-I mean, all of us in our respective departments/units. The doctors with the doctors working there, the interns with the interns working there have to closely communicate. The nurses and midwives also have to communicate among themselves on service delivery and on solving the problems" (ORO-HOSP).

Communication followed a hierarchical path when information or expectations were being transmitted from the top of the system down through the various facility levels, as one participant described: "Orders trickle down from the authorities at the zone level to the ones at the woreda (district) level, then to the health directory, then to the health center, then finally to the health posts. These orders are usually transmitted orally but there are cases when we file them as written forms. We will be given instructions, updates on policies and regulation so we can apply them to our model. We are also given guidance on the best route to implement the reforms, then we inform the community on the steps to be taken" (ORO-HP). It was not clear how information, requests, and expectations were conveyed from the bottom up in this system.

\section{Health extension workers}

HEWs were seen as the key link between mothers in the local community and the entire health system through initial identification during pregnancy and postpartum follow-up. In the health system design, Health Development Army volunteers were supposed to identify pregnant women or recent home births and pass that information to the HEWs. HEWs then performed the critical role of home visits and monitoring mothers and babies in the community. They sometimes managed home delivery issues and follow-up care for preterm, LBW, and sick newborns: "Since the primary responsibility of the health extension worker is within the community in rural [areas], they are accountable to follow newborn and maternal health conditions. Them being there will support us, because they will take the responsibility to ensure pregnant mothers initiate follow-up and come to the health center for childbirth. Then we will follow up, calculate the EDD [estimated date of delivery], and identify those who will come for follow-up visits or not. Further, the health center will decide which newborn case can be treated here and transfer those 
Table 3 Space, power, and water functionality across facility levels

\begin{tabular}{ll}
\hline Facility Level & Problem \\
\hline Space & $\begin{array}{l}\text { Only space for immediate assessment and } \\
\text { referral } \\
\text { Health post }\end{array}$ \\
& $\begin{array}{l}\text { 1-room facilities } \\
\text { No separate space form, mothers, neonates, or }\end{array}$
\end{tabular}
preterm, LBW, and sick newborns

Condition of building compromised (roof caved in)

Lack of fully equipped rooms

Health center

Primary hospital and general hospital
Size of existing rooms inadequate

Could not serve multiple preterm, LBW, and sick newborns who arrived simultaneously

No inpatient or pediatric unit

Could not accommodate newborn when mother was getting care and unable to care for her newborn

Necessary to refer cases beyond first aid due to lack of space

Multiple activities usually combined in 1 or 2 rooms

L\&D shared with postnatal ward; space limited and uncomfortable

No postnatal room; newborns with mothers in waiting room

Preterm newborns cared for in delivery room

No space for parent to rest or sleep when newborn was admitted for care

Antenatal care, family planning, Integrated Child Illness in 1 room; prenatal and pre-labor in another room

No separate room for neonates or preterm, LBW, and sick newborns

Shortage of rooms for delivery

Shortage of space in NICU; had to serve only most critically sick newborns

No space for newborns in delivery rooms

The only space for breastfeeding mothers was in a separate building away from the NICU

Space for doctors and nurses was crowded

KMC room had no sink and limited beds

Delivery room lacked beds; mothers on floor mattresses

Shortage of beds in mini-NICU; preterm, LBW, and sick newborns shared beds

"Laying 4 and 5 kids on 1 bed is very difficult. That is how we are using it. I think that's why our work is not effective. Preterm infants that come [to] us rarely survive because both the septic and the healthy sleep together" (OROHOSP)

KMC babies and mothers in the delivery room with others

Neonatal room did not meet standards

No space for parents in NICU; they had to sleep outdoors or in the latrine
Table 3 Space, power, and water functionality across facility levels (Continued)

\begin{tabular}{ll}
\hline Facility Level & Problem \\
\hline Sometimes had to refer preterm, LBW, and sick \\
newborns due to shortage of beds \\
Multiple babies in 1 incubator \\
Neonates put into adult and surgical wards due \\
to lack of space
\end{tabular}

Power

Health post

Health center

Primary hospital

Power interrupted and unreliable

"There [are times that] the power will be off up to 2 days. Even there was time that we take our patients to other hospital by ambulance due to lack of power" (AA-HOSP)

Generator and/or solar power do not ensure uninterrupted supply Power/generator failure can also result in water failure

Water

Health post

Only periodic water

Health post

Health center

Reliance on river water

No water of any kind whatsoever

Only 3 reported that water was continuous/ uninterrupted, with 1 due to mountain location

Collected and used rain water

Health center

General hospital and specialized hospital

Collected and used unclean river water "We don't have clean tap water. We have to get it from the river. Mind you the kinds of infections and other waterborne diseases that may be caused as a result of this" (ORO-HC)

Collected and used water from a nearby source; not available on-site

Even in a new health center facility, water pipes had leaks; others reported broken pipes

Water came only on alternate days

Mothers not able to clean up after birth "It is difficult for a mother to go home covered in blood after birth. .. For example, if a mother gives birth here on dusty space, then it is no different from giving birth at home" (AMH-HC)

Shortages for 1 to 2 weeks

"We may not get water for 1 or 2 weeks. To eat our food, we have to buy packed water. Even we do not get to wash our hands. We prepared large water container, so we fetch from that. We have to carry from the ground [floor] to the second floor" (AA-HOSP)

Periodic interruptions due to an aged building

Parents restricted from visiting preterm, LBW, and sick newborns in NICU if there was no water to wash visitor gowns

which need a critical follow-up. That is how we do things" (AMH-HC).

HEWs played a critical role in referring preterm, LBW, and sick newborns to higher levels of care, especially in rural areas and especially following home births. Several participants spoke of the need for leadership to recognize this critical role and prioritize it. In some cases, HEWs accompanied patients to higher-level care. 
When women self-referred to higher care, it was seen as a failure of the HEW identification and tracking system.

Participants made several recommendations to improve the HEW role and functionality in the care of preterm, LBW, and sick newborns:

- Conduct initial and ongoing training and capacity building; this is necessary to increase skills if HEWs take on expanded, more sophisticated roles;

- Improve referral system feedback to strengthen the relationship between HEWs and higher-level facilities;

- Increase supervision and support for HEWs;

- Increase governmental support for the role of HEWs and commitment to sustained improvements; and

- Demonstrate leadership respect for HEWs so that others within the larger system adopt a respectful attitude toward HEWs.

Participants also noted that the performance of HEWs could be improved only within the limits of the current system's infrastructure; problems related to the functionality of water, power, and roads would limit even the best trained and supported HEWs.

\section{Health management information systems and data monitoring and evaluation}

Data were being collected at multiple levels throughout the health system, but not consistently within levelsnor even within facilities in some cases. All 3 facility levels-health post, health center, and hospital-reported at least one example of using data for performance monitoring, quality improvement, or health trends. Participants were aware that documentation was important, not only for individual patient tracking but also for health trend tracking, such as observing preterm birth rates, for larger system planning.

\section{Information production}

Data collection practices were problematic on many levels. Primary documentation seemed to be associated with referral, although this may be due to the focus of the interview instrument on referrals. The data collection system was variable, and even erratic, depending on the individual facility and the level of facility. A participant from a health post commented, "Another thing is since we don't have a standard referral form, some information may be left out, which is dangerous" (ORO-HP). Data were recorded on patient cards, log books, booklets, referral forms, and plain letter paper. It was unclear how data were tabulated, collected, and maintained. Health posts played a critical role in gathering data and documenting patient visits. At the kebele (ward) level, data were recorded in 2 places: the booklet of diagnosis guidelines, and the newborn registration log books (for future follow-up by HEWs for antenatal care). Health post staff were aware that proper documentation informed quality supervision and performance monitoring.

\section{Information use}

Ongoing performance monitoring appeared to be happening especially in higher-level facilities based on observation, but not necessarily monitoring data. Several respondents supported the idea of performance monitoring and called for more: "Such things should not be periodic, it should be continuous and the top leaders should design a control system for lower-level implementers. So it is good if it has monitoring and evaluation" (AA-HC). They recognized that performance monitoring could reveal training effectiveness. One urban health center spoke about a strong performance monitoring link between the hospital and health center, and with the FMOH.

\section{Access to essential medicines, supplies, and equipment Equipment functionality and distribution}

Problems with dysfunctional equipment and ineffective equipment distribution systems were widespread. Not only was equipment not being purchased, but when purchases were made without consulting or involving providers, they risked being unusable. In the past, nongovernmental organizations provided support to borrow equipment, but this was not a sustainable solution.

Equipment, materials, and medications were unavailable, expired, or broken at all levels of the system. For the purposes of this report, ambulances are classified as equipment because respondents referred to them as such. Few facility leaders said they were well equipped. Problems at facility levels were as follows:

- Health posts: No available weight scale, timer, breath control, blood pressure monitor, radiant heater, phone network, or ambulance, and incomplete medication supplies. Some equipment was provided by nongovernmental organizations but not by the $\mathrm{FMOH}$; some equipment was broken or very old.

- Health centers: No available oxygen supply, radiant heater, incubator, ambulance, phone network, lab reagents, soap, water, or towels; medications were expired or limited. Almost every health center respondent said that ambulances were one or more of the following: unavailable or delayed, lacked oxygen, relied on motorcycles, or experienced big delays; only one health center said ambulances were not a problem.

- Hospitals: No available infusion syringe pump, portable X-ray, oxygen tubes, pulse oximeter, bag valve mask resuscitator, mechanical ventilator, 
Table 4 Readiness to provide newborn care and preterm, LBW, and sick newborn care

\begin{tabular}{lll}
\hline Facility Unit & $\begin{array}{l}\text { Facility } \\
\text { Level }\end{array}$ & Readiness Challenge \\
\hline $\begin{array}{l}\text { Labor and } \\
\text { delivery }\end{array}$ & All & $\begin{array}{l}\text { Variable across facility levels and between } \\
\text { facility }\end{array}$ \\
& Health & $\begin{array}{l}\text { Delivery room not expected nor equipped to } \\
\text { post }\end{array}$ \\
& $\begin{array}{l}\text { Provide neonatal service } \\
\text { non-emergency patients referred to health } \\
\text { center }\end{array}$ \\
& $\begin{array}{l}\text { Lacked qualified providers for newborn care } \\
\text { Health } \\
\text { center }\end{array}$ & $\begin{array}{l}\text { Provided care in L\&D but only had } 1 \text { room and } \\
\text { needed more space }\end{array}$
\end{tabular}

Provided the service at the time needed but space and comfort were inadequate

Equipped for neonatal care and for preterm, LBW, and sick newborns, but health center rarely encountered them

Served routine-care neonates but not preterm LBW, and sick newborns; no materials or specialized provider

Had laboratory technician and new rooms but lacked additional professionals

Prepared, but preterm, LBW, and sick newborns transferred to under-5 department or hospital as needed

Space limitations prohibited neonatal care in L\&D

Inadequate supplies, services, and space for preterm, LBW, and sick newborn care

Hospital Not enough training for L\&D providers to give proper neonatal care

May not have had space for neonates or proper equipment to give care

Hospital staff was ready but lacked ventilator

Hospital lacked space and beds; could not accommodate newborns

Lack of infrastructure, equipment, and food for patients hindered abilities

L\&D rooms lacked handwashing stations

NICU All Most respondents reported no NICU; no expectation for that to change

Health Never expected

post

Health No NICU

center

Hospital Needed supplies and additional providers

Needed space for parents; no place for them to wait or sleep

Material shortage, including of medication and equipment

NICU isolated; parents could look through glass; mother could visit if baby improved; when no water to clean gowns, parents could not enter $\mathrm{NICU}$
Table 4 Readiness to provide newborn care and preterm, LBW, and sick newborn care (Continued)

\begin{tabular}{|c|c|c|}
\hline Facility Unit & $\begin{array}{l}\text { Facility } \\
\text { Level }\end{array}$ & Readiness Challenge \\
\hline \multirow[t]{6}{*}{$\overline{K M C}$} & All & $\begin{array}{l}\text { Adequate in many facilities; most offered KMC } \\
\text { even if referring up }\end{array}$ \\
\hline & \multirow[t]{2}{*}{$\begin{array}{l}\text { Health } \\
\text { post }\end{array}$} & $\begin{array}{l}\text { KMC service provided prior to referral to health } \\
\text { center }\end{array}$ \\
\hline & & $\begin{array}{l}\text { Space may be just a corner or nook or no } \\
\text { space at all }\end{array}$ \\
\hline & $\begin{array}{l}\text { Health } \\
\text { center }\end{array}$ & $\begin{array}{l}\text { No separate room; KMC in } L \& D \text { or pediatrics; } \\
\text { little space with no food storage }\end{array}$ \\
\hline & \multirow[t]{2}{*}{ Hospital } & $\begin{array}{l}\text { KMC and NICU joined in this hospital; } \\
\text { sophisticated care available, including water } \\
\text { and toilet }\end{array}$ \\
\hline & & $\begin{array}{l}\text { KMC room available for dyads when mother } \\
\text { was healthy; when mother admitted, baby } \\
\text { stayed in KMC without parent }\end{array}$ \\
\hline \multirow[t]{3}{*}{$\begin{array}{l}\text { Inpatient } \\
\text { ward }\end{array}$} & $\begin{array}{l}\text { Health } \\
\text { post }\end{array}$ & Never expected \\
\hline & $\begin{array}{l}\text { Health } \\
\text { center }\end{array}$ & None; accommodated elsewhere in facility \\
\hline & Hospital & $\begin{array}{l}\text { Facility with NICU could care for preterm, LBW, } \\
\text { and sick newborns; budgets may not have } \\
\text { covered basics like diapers, clothes, or even } \\
\text { necessary treatment for preterm, LBW, and sick } \\
\text { newborns }\end{array}$ \\
\hline
\end{tabular}

Abbreviations: $K M C$ kangaroo mother care, $L \& D$ labor and delivery, $L B W$ low birth weight, NICU neonatal intensive care unit

continuous positive airway pressure, incubator, phone, diapers, baby clothes, or personal protective equipment for staff exposed to radiation and infection. Newborns were exposed to infection by sharing beds, incubators, and phototherapy machines. There were shortages of ambulances and NICU supplies, such as infusion syringe pumps, nasal prongs for preterm, bag valve mask resuscitators, vitamin $\mathrm{K}$, and glucometers.

\section{Health financing}

Respondents were nearly unanimous that there was no sustained financial support for preterm, LBW, and sick newborn care in facilities. Hospital leaders talked about being able to include it in the general budget, but they asserted that there was no separate funding support for programs for preterm, LBW, and sick newborns. One health center representative observed: "I think there needs to be a properly budgeted program which rigorously works to increase awareness in the society. Simultaneously, the health center needs to support facilities and even ambulances so that it can provide the level of health care service promised to the community" (ORO$\mathrm{HC}$ ). Funding needs at health centers and hospitals included: specialist salaries, printing of guidelines and policies, basic sick baby supplies (e.g., milk and clothing), 
transportation for low-income patients (other than transfer ambulances), food for patients and parents, phone service for providers. In the case of health posts, earmarked funds are necessary if there were no actual programs for the care of preterm, LBW, and sick newborns.

\section{Leadership and governance \\ Guidelines, policies, and protocols}

Participants were asked a series of questions about guidelines, clinical standards, protocols, or training modules relevant to prevention, management, or care of preterm, LBW, and sick newborns-specific to the level of facility they supervised. Responses were analyzed to assess how the facility leadership prioritized use of guidelines and to what degree guidelines were available and being used. One big challenge was that leadership across facilities interpreted the concepts of guidelines, policies, and manuals differently. At health posts, references to guidelines primarily included training manuals and chart booklets, while at health centers and hospitals, guidelines might include published manuals, guidelines, and protocols. Availability of guidelines varied widely across facility levels, within the same level but between facilities, and even within facilities. There was no consistency anywhere in the system regarding guidelines for care of preterm, LBW, and sick newborns. Perhaps the most widely available guidelines were related to referrals, some of which were very simplified at the health post level and accessible in chart booklets. This is not to say that other guidelines did not exist, but they were not universally available. Most importantly, if newborn guidelines were available, they were not specific to preterm, LBW, and sick newborns.

Use of guidelines depended primarily upon availability. When guidelines were available, they were seen as an important resource: "We provide the guideline for the assigned provider at the unit and we make sure the guideline stays in the unit; we even have a locker for that so to make sure anybody else assigned at that unit can access the guideline. Because we can't work without the guideline, the guideline is required critically. The guideline and the log book are inseparable documents" (AMH-HP). Guidelines were used for assessment and referral, resuscitation, parent access to baby, complicated case management, and treatment. One health center representative spoke about posting key points from the guidelines in the medical compound for easy reference. Some spoke about using outdated guidelines because they saw that as better than no guidelines at all. Nearly all those who were aware of, and knowledgeable about, guidelines and policies found them clear and understandable.

For those who did not use or adhere to the guidelines, across all facility levels, reasons ranged from poor dissemination to outdatedness. Respondents recognized the need for ongoing guideline dissemination: "These materials-according to the system, it could be from the ministry of health to the health bureau to the sub-cities and woredas - should flow to us timely. And the updates should be properly introduced and training the providers is needed to introduce the providers with new materials so that they can primarily focus on the programs" (AA$\mathrm{HC}$ ). When asked about what might facilitate expanded usage, participants had several key suggestions. Challenges and facilitators for guideline use are in Table 5.

\section{Supervision and feedback}

Staff supervision and feedback were valued by participants, but responses suggested the feedback needs to be systematized and bolstered at all levels across and within facilities. Feedback was primarily through patient referrals. Feedback at catchment meetings was recognized as

Table 5 Guidelines for care of preterm, LBW, and sick newborns: challenges and facilitators to provider use

\begin{tabular}{|c|c|}
\hline Challenge & Facilitator \\
\hline $\begin{array}{l}\text { Lack of adequate dissemination; often disseminated to individuals rather than } \\
\text { facility units; providers removed guidelines from facility for personal use }\end{array}$ & $\begin{array}{l}\text { Fast dissemination; suggest using schools for dissemination; } \\
\text { dissemination to facility units; leaflets and flyers as distribution } \\
\text { materials }\end{array}$ \\
\hline Lack of updated guidelines & Timely updates: publication and via internet \\
\hline Guidelines did not match well to professional specialty or skill level & $\begin{array}{l}\text { Complex cases require guidelines for treatment; Guidelines promotes } \\
\text { adherence }\end{array}$ \\
\hline $\begin{array}{l}\text { Lack of staff knowledge, which may also have manifested as resistance to } \\
\text { policies }\end{array}$ & Performance monitoring and feedback to staff not using guidelines \\
\hline Lack of supplies, equipment, and infrastructure renders guidelines unusable & Equipment provision for the delivery of care \\
\hline Lack of periodic professional training & Ongoing training, including in-service \\
\hline \multicolumn{2}{|l|}{ Staff "too busy" to follow manuals; work overload } \\
\hline $\begin{array}{l}\text { Lack of relevance to community needs especially at lower-level health } \\
\text { facilities }\end{array}$ & \\
\hline
\end{tabular}


an opportunity to provide guidance on areas for improvement as well as recognition of accomplishments from the hospital to the health center. Similarly, feedback between health posts and Health Development Army leaders offered opportunities to reduce the number of out-of-facility births. Supportive supervision and mentorship were valued but appeared to come primarily from external sources rather than from direct supervisors or leadership. More stringent supervision and mentoring were needed universally, and especially in NICUs, where highfunctioning staff were critical. At the health post level, participants requested that district-level supervisors support health posts when supporting programs from tangential sectors such as agriculture, and also, that they provide follow-up supervision and feedback after trainings.

\section{Leadership priorities}

Participants were in nearly universal agreement that maternal and child health was a focus priority for the $\mathrm{FMOH}$, citing evidence that emphasis was placed on NICU training, referral, and transport issues. The link between hospitals and health centers helped to maintain the maternal and child health focus. For maternal and child health to be considered a priority focus, however, the $\mathrm{FMOH}$ would need to secure additional funding support (including funds to meet newborns' needs); establish an organized program for preterm, LBW, and sick newborns; and ensure that leadership would drive the program forward. Although respondents noted that treatment and management of preterm, LBW, and sick newborns were considered FMOH priorities, a great gulf remained between stated priorities and availability of materials, recommendations, and training to implement programs. As one respondent stated, "The government has prioritized maternal and child services by recognizing preterm or LBW newborns' need for early treatment and management, but we are not providing all necessary services here for newborns. .. For the health center to improve service, there should be a protocol; we will get confused to treat or manage such cases in current situations. We will be more confident when necessary materials are adequately available. .. In addition, training should be provided to professionals because knowledge is important for service. Knowledge leads to skill" (AMH-HC).

\section{Community engagement}

Community engagement is not one of the WHO health systems building blocks, but it is vital to providing ongoing care. Participants reported that community engagement and awareness were accomplished through key individuals in the communities: Health Development Army volunteers, religious leaders, traditional birth attendants, and midwives. As one leader suggested, "We should select some influential family members and community members and make them part of trainings to disseminate the information to the community through the community members" (AMH-HP). Additionally, providers recognized that responding to extended families in hospital settings and relying on women who had successfully delivered in facilities were informal ways to spread goodwill between the health system and community. As one health center participant explained, "The most difficult thing for us is that women seem to believe and rely on what they talk about amongst themselves rather than what we teach them. For them we are regarded as outsiders, so we try to make those women from the community who have had health care services speak out on our behalf, and it has proved to be successful" (ORO$\mathrm{HC}$ ). Engagement was seen as particularly difficult when the community was mobile. Topics recommended for community awareness included home birth risks, importance of medical care for newborns, and family planning. Participants also acknowledged that leaders must allocate budgets to create awareness.

Some staff at facilities described efforts to engage the community by showing respect for cultural traditions, like coffee ceremonies and porridge making, and by observing Neonatal Day as a holiday. These efforts to draw mothers and families into the health system were up against the strong pull of traditions that limit access to facilities, including prohibiting mothers from leaving home for 40 days following birth, and concerns about babies or mothers dying in a facility far from home.

Communication with families was recognized as vital to health system readiness to serve families of preterm, LBW, and sick newborns. Participants reported that providers' communication with families in clinical settings varied widely depending on whether responding to their questions or providing updates or information. Communication may have varied widely also by facility type or site. Table 6 shows how respondents saw communication playing out between providers, facilities, and families.

\section{Programs for preterm, LBW, and sick newborns: existence and components}

Participants were asked about the existence and components of programs for preterm, LBW, and sick newborns. Most respondents said their facility had no program of which they were aware. Health post leaders mentioned programs for referral and postnatal education, and health center personnel mentioned equipment (heater, ambulance, and booklets), supervision, the referral process, and previously existing programs. Hospital leaders discussed complex medical programs they currently had. All respondents recognized the importance of focused programs for 
Table 6 Communication between families and facility staff: domains and subdomains

\begin{tabular}{ll}
\hline Domains & Subdomains \\
\hline Updating mother/father, sometimes family & Updating done frequently but not during emergency procedures \\
& Recognition that good updating was part of compassionate and respectful care \\
& Communicating bad news to parents could be very challenging \\
& $\begin{array}{l}\text { Responsibility for updating parents varied by specialist, general practitioner, or nurse, } \\
\text { depending on who was attending to the baby }\end{array}$ \\
Parent/family questions policy & $\begin{array}{l}\text { Parents were encouraged to ask about newborns anytime; questions from extended } \\
\text { family were also answered on demand }\end{array}$ \\
Hospitals and health centers established some formal lines of & $\begin{array}{l}\text { Where policies existed about communicating baby status to parents, they were } \\
\text { usually followed; some facilities had no policy }\end{array}$ \\
Communication with patients & $\begin{array}{l}\text { Midwives were responsible for educating inpatients about postnatal care, newborn } \\
\text { care, family planning, etc. }\end{array}$
\end{tabular}

preterm, LBW, and sick newborns and requested support. As one person said, "First the programs should be initiated; I don't think the programs are beyond paper" (AMH-HC). Another called for a coordinated effort: "First, there should be trained personnel, then materials that help to provide services for newborns should be adequately available including medications. .. moreover, this program should have a focal person who works on maternal and child health. .. and a strengthened follow-up system must be placed. I think different stakeholders including woreda, zone health departments, and partner organizations must be involved to provide regular support" (AMH-HP).

\section{FMOH support for preterm, LBW, and sick newborn programs}

The FMOH has clearly stated that maternal and child health is a public health priority, but that has not positively affected staff strengthening, funding, or materials provision that would support initiating or continuing programs for preterm, LBW, and sick newborns. Respondents unanimously agreed that no funds were available from the FMOH for initiating any kind of program for preterm, LBW, and sick newborns at any facilities. Similarly, no respondents were aware of any staff-related support for preterm, LBW, and sick newborn program activities, with the exception of limited training opportunities. Responses were nearly unanimous that there was no support for materials or training guides. A few people referred to initial training or booklets they had received, but they were primarily for midwifery training on newborn care rather than care for preterm, LBW, and sick newborns.

The lack of sustained support for preterm, LBW, and sick newborn programs created a reliance on nongovernmental organizations to fill that gap. The FMOH has not consistently supplied equipment or guides to support ongoing preterm, LBW, and sick newborn programs. Heaters have been supplied to some facilities, but there was also a call for newborn clothing and medications. One person referred to the unsustainable practice of borrowing equipment, and others described equipment that may have gone unused due to lack of training. Table 7 presents key findings and recommendations.

\section{Discussion}

This qualitative study assessed Ethiopia's health system readiness to support public health facility care of preterm, LBW, and sick newborns. Each of the WHO health system building blocks were considered, and findings revealed that public hospitals, health centers, and health posts faced significant facility and system challenges in all 7 components, making optimum care for preterm, LBW, and sick newborns difficult. Unlike other studies looking at multiple countries, in this study we gathered in-depth information across 3 economically and geographically diverse regions of one country. The findings from this study provide evidence to inform action steps to address complex system-level challenges.

Over the past decade, WHO and others have given much attention to health systems strengthening [7, 15], and the Ethiopian government has reaffirmed commitments and consolidated gains [22]. We relied on facility leadership - the immediate recipients of health system inputs to facilities - as our main data source, building on findings from other studies that health care professionals are key information sources to monitor progress in health systems $[23,24]$.

\section{Health service delivery infrastructure and readiness}

Ethiopia has achieved significant expansion of all levels of public health facilities in the last 2 decades [17]. Facilities are reported to be well equipped to provide care, but most cited problems in one or more critical areas that inhibited effective provision of care. Reliable power 
Table 7 Key findings and recommendations related to World Health Organization building blocks

\begin{tabular}{|c|c|}
\hline WHO Building Block & Key Finding \\
\hline Service delivery & $\begin{array}{l}\text { - Lack of reliable power and water across facility levels } \\
\text { - Lack of space for preterm, LBW, and sick newborns and the } \\
\text { mothers }\end{array}$ \\
\hline $\begin{array}{l}\text { Health workforce } \\
\text { and human } \\
\text { resources }\end{array}$ & $\begin{array}{l}\text { - Shortage of adequate and well-trained health professionals } \\
\text { of almost every category at all levels of health facilities } \\
\text { - Neonatologists/pediatricians and neonatal nurse specialists } \\
\text { in the country were few in number and concentrated in } \\
\text { tertiary centers } \\
\text { - Trainings and national programs supporting them were } \\
\text { integrated with general newborn care and not specifically } \\
\text { focused on preterm, LBW, and sick newborn care }\end{array}$ \\
\hline
\end{tabular}

Health management $\cdot$ M\&E data were collected at various levels within the health information systems system inconsistently and irregularly and M\&E

- Available data were not always used for performance monitoring and quality improvement

Access to essential medicines, supplies, equipment

Financing

Leadership and governance

Community engagement appeared to come primarily from external sources rather
- Shortages of medical supplies, equipment and essential medications were widespread in health facilities at all levels - They were unavailable, broken, or inappropriate for use

- Funding allocated for system readiness to care for preterm, LBW, and sick newborns was needed in nearly all facilities at all levels

- Specific funding needs included specialist salaries, printing of guidelines and policies, basic sick baby supplies (e.g., milk and clothing), transportation for low-income patients (other than transfer ambulances), food for patients and parents, phone service for providers.

- Gap in the availability of guidelines and protocols specifically targeting preterm, LBW, and sick newborns

- Staff supervision and feedback were valued by participants, but responses suggested they need to be systematized and bolstered at all levels across and within facilities

- Supportive supervision and mentorship were valued but than from direct facility leadership

- Key facilitators and information disseminators/influencers identified in the study were the Health Development Army, community and religious leaders, and mothers and families who had positive experiences or outcomes of care

- Showing respect for the community's traditions was recognized as an effort to positively change the perception of the community

\section{Recommendation}

- Create separate building/rooms specially designed for

preterm, LBW, and sick newborn care, with spaces for mothers/ caretakers

- Have backup power source (generator)

- Have a water reservoir

- Provide continuous workforce training, motivation, and support to boost skills and commitment in the face of a highly demanding environment of intensive and advance newborn care

- Address health care workforce shortages within facilities to meet adequate staffing levels to provide the necessary labor-intensive inpatient care for newborn

- Continue to recognize and support health extension workers

- Improve the consistency and quality of data collection and use of data at all levels of health system

- Prioritize procurement and maintenance of critical supplies and equipment

- Fund a program specifically to support preterm, LBW, and sick newborn care at the facility level, which may help to alleviate challenges and ultimately improve the available care

- Develop evidence-based, up-to-date guidelines and protocols specific to the care of preterm, LBW, and sick newborns, and communicate them across the system tier with appropriate supervision

- Develop more stringent supervision and mentoring, especially in NICUs where high-functioning staff are critical

- Improve awareness through health education, peer modeling, and dissemination of good experiences

Abbreviations: LBW low birth weight, M\&E monitoring and evaluation, NICU neonatal intensive care unit, WHO World Health Organization

and water have remained major problems, especially in lower-level facilities [25]. Space in health facilities for newborns in need of special care, including space for mothers to stay with their babies, was either absent, inadequate, or lacked privacy. Similar problems were reported in other African and Asian countries [9].

\section{Workforce/human resources}

Health workforce shortages were found to be a critical obstacle to delivering quality care for preterm, LBW, and sick newborns. As found in other reports [8, 9], nationwide shortages of adequate and well-trained health professionals of almost every category were found at all levels of health facilities. The problem was worse in rural and remote regions of the country and at the lowest levels of the health system. The number of neonatologists, pediatricians, and neonatal nurse specialists in the country were few and concentrated in tertiary centers [26]. This problem offered 2 equally important solutions: produce more graduates, and continuously strengthen the capacity of existing staff working in remote and lower-level health facilities. Trainings and the national programs supporting them were integrated with general newborn care and not specifically focused on preterm, LBW, and sick newborn care [27]. Findings also emphasized the important role of HEWs in 
identifying and referring preterm, LBW, and sick newborns, and the need for recognition and continuous support of HEWs.

\section{Supplies, equipment, and essential medications}

Shortages of medical supplies, equipment, and essential medications were a widespread problem in health facilities at all levels. They were often unavailable, broken, or inappropriate for use. In another report, fewer than half of all facilities had most supplies and equipment needed for preterm, LBW, and sick newborns, and these facilities were primarily specialized hospitals rather than general and primary hospitals [28]. In another report, $35 \%$ of Ethiopian health facilities experienced stock-outs of essential medicines, and approximately $8 \%$ of medicines in stock had expired [29].

\section{Health financing}

This study showed that specific funding allocated for system readiness to care for preterm, LBW, and sick newborns was needed in nearly all facilities at all levels. For families, the birth of preterm, LBW, and sick newborns could be financially catastrophic. Shifting from a reliance on out-of-pocket payment to prepayment and risk pooling is a critical part of the health financing transition that most countries go through as they get richer. The Ethiopia health care financing strategy aims to replace user fees with funding from the central government. This is expected to boost utilization of services and consequently increase demand on resources [30]. The health care financing strategy targets reduction of out-of-pocket expenditures for direct clinical care services costs, however, and does not include indirect costs for supplies in the form of clothing and formula milk for feeding (when indicated) and emergency transportation costs-which is important for saving lives in cases of emergency by facilitating travel to health facilities.

\section{Leadership and governance}

The availability of guidelines, policies, and protocols, and the practice of supervision and feedback were used as measures of effective leadership and governance. There was a notable gap in the availability of guidelines and protocols specifically targeting preterm, LBW, and sick newborns. From a recent analysis of health system bottlenecks, policy documents in circulation among senior officials were not disseminated to lower-level managers who did not always adhere to guidelines for special newborn care [9]. This study, too, found that in Ethiopia, narrow dissemination and outdated materials contributed to poor adherence to national protocols and guidelines. Another study in Ethiopia reported that hospital activities were poorly supported with a lack of updated or appropriate guidelines and protocols [31].
Staff supervision and feedback were valued by participants, but responses suggested they need to be systematized and bolstered at all levels across and within facilities. Most feedback was related to referrals, and participants spoke positively about effective systems between facilities, especially case-specific feedback. Functional referral systems are crucial for the referral of all patients who need care that cannot be provided in the health facility. An essential component of quality of care for preterm, LBW, and sick newborns is proper handover to the receiving facility and feedback to the sending facility when completing a referral. This is a global standard for improving quality of service for preterm, LBW, and sick newborns [32].

Supportive supervision and mentorship were valued but appeared to come primarily from external sources rather than from direct facility leadership. More stringent supervision and mentoring were suggested universally and especially in NICUs where high-functioning staff are critical. In a study in India, nursing staff felt that there were not enough proficient providers to mentor the nurses to safely perform skills, and they identified lack of mentorship as an obstacle for improving service delivery for preterm, LBW, and sick newborns [33]. Ongoing on-site training, mentoring, and supervision were also cited as absolutely necessary to maintaining the knowledge and motivation of mid-level health care providers and reducing neonatal mortality $[34,35]$.

Ethiopia's National Neonatal and Child Survival Strategy aims to reduce the neonatal mortality rate from 28 deaths per 1000 live births to 11 deaths per 1000 live births [6]. Although some interventions like KMC, which were defined in this strategy, may have benefited preterm, LBW, and sick newborns, existing programs were not specifically focused on supporting the care of such newborns in all levels of health facilities.

\section{Data monitoring and evaluation/health management information systems}

This study showed inconsistent and irregular monitoring and evaluation data collection at different levels within the health system. Available data were not always used for performance monitoring and quality improvement. The use of health management information systems is important in developing health care services, but many LMICs struggle with data quality problems resulting in incomplete, inaccurate, and untimely information, which is not useful for health decision making [36]. In Ethiopia where the health management information system is the major source of health information [37], the overall data quality was found to be below international standards $[38,39]$. These findings also showed the need for improvements in data quality and usefulness. 


\section{Community engagement}

This study revealed that community engagement was considered an important component of serving families with preterm, LBW, and sick newborns. The key facilitators, information disseminators, and influencers identified in the study were the Health Development Army, community and religious leaders, and mothers and families who had had positive experiences or outcomes of care. Showing respect for the community's traditions was recognized as an effort to positively change the perception of the community. Community engagement in a mobile society was found to be a challenge. The findings were in line with the FMOH strategy to reduce overall neonatal mortality [6]. While engaging community in the care of preterm, LBW, and sick newborns is reported as a major obstacle in many countries [9], a recent study in Malawi showed a shift in perception of community members through health counseling, peer modeling, and personal success with KMC [40].

\section{Limitations}

The input from facility leaders reflects their experiences and expectations, and possibly their optimism or frustration. Expanding the sources of information about the functioning of the health system was beyond our resources. Additional evidence is available from existing hospital data, central reporting systems, and central government representatives. The proportion of providers in facility leadership roles who participated in the interviews may not be representative of all clinicians and providers in the facilities. Information obtained from interviews was not verified by observation.

\section{Conclusion}

The Ethiopian health system has challenges in terms of its readiness to support health facilities to provide quality care for preterm, LBW, and sick newborns. Challenges were reported in all 7 WHO health system building blocks-leadership and governance, health financing, health workforce, essential medical products and technologies, health service delivery, health management information system, and community partnership. Many of the readiness challenges across the health system seem to stem from the inadequate emphasis given to the special care required for preterm, LBW, and sick newborns.

\section{Recommendations}

Based on the findings of this study, we propose the following recommendations and suggested actions (see Table 8).
Table 8 Recommendations and actions to improve health system readiness for preterm, LBW, and sick newborns

\begin{tabular}{|c|c|c|}
\hline $\begin{array}{l}\text { Health System } \\
\text { Building } \\
\text { Blocks }\end{array}$ & Recommendations & Suggested Actions \\
\hline $\begin{array}{l}\text { Service } \\
\text { delivery/ } \\
\text { infrastructure } \\
\text { Supplies/ } \\
\text { equipment }\end{array}$ & $\begin{array}{l}\text { - Consideration of critical } \\
\text { infrastructure (space, power, } \\
\text { water) and equipment to } \\
\text { meet the specific needs of } \\
\text { preterm, LBW, and sick } \\
\text { newborn care }\end{array}$ & $\begin{array}{l}\text { - Create separate building/ } \\
\text { rooms specially designed } \\
\text { for preterm, LBW, and sick } \\
\text { newborn care with space } \\
\text { for mothers/caretakers } \\
\text { - Have backup power } \\
\text { source (generator) } \\
\text { - Have a water reservoir } \\
\text { - Prioritize procurement and } \\
\text { maintenance of critical } \\
\text { supplies and equipment }\end{array}$ \\
\hline Workforce & $\begin{array}{l}\text { - Continuous training, } \\
\text { motivation, and support for } \\
\text { the workforce are necessary } \\
\text { to boost skills and } \\
\text { commitment in a highly } \\
\text { demanding environment of } \\
\text { intensive and advance } \\
\text { newborn care } \\
\text { - Addressing health care } \\
\text { workforce shortages within } \\
\text { facilities to meet adequate } \\
\text { staffing levels for labor- } \\
\text { intensive inpatient care for } \\
\text { newborns }\end{array}$ & $\begin{array}{l}\text { - Train adequate number of } \\
\text { doctors and nurses with } \\
\text { neonatal care skills } \\
\text { - Conduct refresher training } \\
\text { for health workers } \\
\text { working in neonatal units } \\
\text { - Develop strategies to } \\
\text { appropriately remunerate } \\
\text { and incentivize neonatal } \\
\text { health workers } \\
\text { - Provide regular supportive } \\
\text { supervision } \\
\text { - Recognize the role of } \\
\text { health extension workers }\end{array}$ \\
\hline $\begin{array}{l}\text { Governance/ } \\
\text { leadership }\end{array}$ & $\begin{array}{l}\text { - Evidence-based, up-to-date } \\
\text { guidelines and protocols } \\
\text { specific to the care of pre- } \\
\text { term, LBW, and sick new- } \\
\text { borns should be developed } \\
\text { and communicated across } \\
\text { the system tiers with appro- } \\
\text { priate supervision }\end{array}$ & $\begin{array}{l}\text { - Develop guidelines and } \\
\text { protocols that are specific } \\
\text { to preterm, LBW, and sick } \\
\text { newborn care and that } \\
\text { match skill levels of health } \\
\text { workers } \\
\text { - Update the guidelines in a } \\
\text { timely manner } \\
\text { - Disseminate the } \\
\text { guidelines to all facilities } \\
\text { caring for preterm, LBW, } \\
\text { and sick newborns }\end{array}$ \\
\hline $\begin{array}{l}\text { Health } \\
\text { financing }\end{array}$ & $\begin{array}{l}\text { - A program specifically } \\
\text { supporting and funding } \\
\text { preterm, LBW, and sick } \\
\text { newborn care at the facility } \\
\text { level may help to alleviate } \\
\text { the challenges and } \\
\text { ultimately improve the } \\
\text { available care }\end{array}$ & $\begin{array}{l}\text { - Develop sustainable } \\
\text { source of funding } \\
\text { earmarked for preterm, } \\
\text { LBW, and sick newborn } \\
\text { care at the facility level } \\
\text { - Develop mechanisms for } \\
\text { incorporating baby food } \\
\text { and clothing in medical } \\
\text { supplies }\end{array}$ \\
\hline $\begin{array}{l}\text { Data M\&E/ } \\
\text { health } \\
\text { information } \\
\text { system }\end{array}$ & $\begin{array}{l}\text { - Improve the consistency and } \\
\text { quality of data collection } \\
\text { and use at all levels of } \\
\text { health system }\end{array}$ & $\begin{array}{l}\text { - Improve data collection } \\
\text { and storage through } \\
\text { continuous training and } \\
\text { supervision } \\
\text { - Monitor data quality } \\
\text { through regular feedback } \\
\text { and follow-up } \\
\text { - Improve data usage at all } \\
\text { levels of health system }\end{array}$ \\
\hline $\begin{array}{l}\text { Community } \\
\text { engagement }\end{array}$ & $\begin{array}{l}\text { - Increase community } \\
\text { engagement through } \\
\text { awareness creation }\end{array}$ & $\begin{array}{l}\text { - Improve awareness } \\
\text { through health education, } \\
\text { peer modeling, and } \\
\text { dissemination of positive } \\
\text { experience } \\
\text { - Improve family experience } \\
\text { at health facilities } \\
\text { - Create culture-responsive } \\
\text { health care environment }\end{array}$ \\
\hline
\end{tabular}

Abbreviations: $L B W$ low birth weight, M\&E monitoring and evaluation 


\section{Supplementary information}

Supplementary information accompanies this paper at https://doi.org/10. 1186/s12913-019-4672-2.

Additional file 1. Key Informant Interview Guide Facility Leadership.doc qualitative interview instrument.

\section{Abbreviations}

FMOH: Federal Ministry of Health; HEW: Heath extension worker; HMIS: Health management information system; KMC: Kangaroo mother care; L\&D: Labor and delivery; LBW: Low birth weight; LMICs: Low- and middleincome countries; M\&E: Monitoring and evaluation; NICU: Neonatal intensive care unit; USAID: United States Agency for International Development (USAID); WHO: World Health Organization

\section{Acknowledgements}

The authors wish to acknowledge the regional health authorities of Oromia, Amhara, and Addis Ababa regions and the facility leadership in the study facilities, without whose cooperation this study would have been impossible. The Every Preemie-SCALE Ethiopia Implementation Research Collaboration Group includes:

Hagos Gidey, Mekelle University, Mekelle, Ethiopia.

Tedros Hailu, Mekelle University, Mekelle, Ethiopia.

Solomie Jebessa, St. Paul's Hospital Millennium Medical College, Addis Ababa, Ethiopia.

Amaha Kahsay, Mekelle University, Mekelle, Ethiopia.

Kemal A. Kuti, Madda Walabu University, Robe, Ethiopia.

Gillian A. Levine, Global Alliance to Prevent Prematurity and Stillbirth (GAPPS), Lynnwood, WA, USA.

Judith Robb-McCord, Project Concern International, Washington DC, USA Alemu Tesfahun, Defence University, College of Health Sciences, Ethiopia. Berhe Dessalegn Tuamay, College of Medicine and Sciences, Adigrat University, Ethiopia.

\section{Authors' contributions}

$J L$ conceived of the overarching study. $J L$ and WG served as principle investigators. JL, AT, EW designed the study, carried out the research, and coordinated all aspects of the study. WG and JR assisted in study design. WG assisted in the implementation of the study. AU, EW, YT, AT, AY, KS and JL analyzed and synthesized the data, and wrote the manuscript. Research Study Groups members from Ethiopia assisted in the code book development and data synthesis. All authors read and approved the final manuscript.

\section{Funding}

This study was made possible by the generous support of the American people through the United States Agency for International Development (USAID), under the terms of the Cooperative Agreement AID-OAA-A-1400049. The contents are the responsibility of the authors and do not neces sarily reflect the views of USAID or the United States Government. The funders had no role in the study design, data collection and analysis, decision to publish, or preparation of the manuscript. Additional support was provided by the Global Alliance to Prevent Prematurity and Stillbirth (GAPPS) to complete the manuscript and publication process.

\section{Availability of data and materials}

The qualitative data, individual stories and narratives have been collected in personal circumstances. Informants were assured that their contribution will remain confidential to the research project and will not be shared.

\section{Ethics approval and consent to participate}

Potentially eligible and interested participants received consent forms in their preferred language and were informed that their participation would be voluntary, and there would be no professional or personal consequences or benefits to participation. Informed written consent was obtained from all participants. To avoid possible coercion, no financial incentives were provided. This study was reviewed and approved by the institutional review boards of the St. Paul's Hospital Millennium Medical College, Addis Ababa, Ethiopia, (IRB No. PM23/111) and Project Concern International (IRB No. 25). Letters of support were secured by the Global Alliance to Prevent Prematurity and Stillbirth (GAPPS) from all institutions and offices where data were collected.

\section{Consent for publication}

Not applicable.

\section{Competing interests}

The authors declare that they have no competing interests.

\section{Author details}

${ }^{1}$ Madda Walabu University, Bale Robe, Ethiopia. ${ }^{2}$ Wolaita Sodo University, Wolaita Sodo, Ethiopia. ${ }^{3}$ Federal Ministry of Health, Addis Ababa, Ethiopia. ${ }^{4}$ St. Paul's Hospital Millennium Medical College, Addis Ababa, Ethiopia. ${ }^{5}$ Global Alliance to Prevent Prematurity and Stillbirth (GAPPS), 19009 33rd Avenue W, Suite 200, Lynnwood, WA 98036, USA.

Received: 19 July 2019 Accepted: 23 October 2019

Published online: 21 November 2019

\section{References}

1. Chawanpaiboon S, Vogel JP, Moller A, Alkema L. Global, regional, and national estimates of levels of preterm birth in 2014: a systematic review and modelling analysis. Lancet Glob Health. 2019;7:e37-46. https://doi.org/ 10.1016/S2214-109X(18)30451-0.

2. World Health Organization. Global Health Observatory (GHO) data. 2017. https://www.who.int/gho/child_health/mortality/causes/en/. Accessed 22 January 2019.

3. United Nations Children's Fund (UNICEF), World Health Organization; The World Bank; United Nations, Department of Economic and Social Affairs, Population Division; United Nations Economic Commission for Latin America and the Caribbean Population Division. In: Levels and trends in child mortality: Report 2013. New York, NY: UNICEF; 2013. https://www.who. int/maternal_child_adolescent/documents/levels_trends_child_mortality_2 013.pdf?ua=1. Accessed 25 Mar 2019.

4. Liu L, Oza S, Hogan D, et al. Global, regional, and national causes of child mortality in 2000-15: An updated systematic analysis with implications for the sustainable development Goals. Lancet. 2016;388(10063):3027-35. https://doi.org/10.1016/S0140-6736(16)31593.

5. United Nations Interagency Group for Child Mortality Estimation, and UNICEF. Levels \& trends in child mortality: report 2017: estimates developed by the UN Inter-Agency Group for Child Mortality Estimation. New York: UNICEF; 2017.

6. Maternal and Child Health Directorate, Federal Ministry of Health (FMOH), Federal Democratic Republic of Ethiopia. National strategy for newborn and child survival in Ethiopia, 2015/16-2019/20. Addis Ababa, Ethiopia: FMOH; 2015. http://www.moh.gov.et/sl/web/guest/-/national-strategy-for-newbornand-child-survival-in-ethiopia-2015-16-2019-20-?inheritRedirect=true. Accessed 25 Mar 2019

7. World Health Organization (WHO). Monitoring the building blocks of health systems: a handbook of indicators and their measurement strategies. Geneva: WHO; 2010. https://www.who.int/healthinfo/systems/WHO_ MBHSS_2010_full_web.pdf. Accessed 25 Mar 2019

8. Dickson KE, Simen-Kapeu A, Kinney MV, Lancet Every Newborn Study Group, et al. Every newborn: health-systems bottlenecks and strategies to accelerate scale-up in countries. Lancet. 2014;384(9941):438-54 https://doi. org/10.1016/S0140-6736(14)60582-1

9. Moxon SG, Lawn JE, Dickson KE, et al. Inpatient care of small and sick newborns: a multi-country analysis of health system bottlenecks and potential solutions. BMC Pregnancy Childbirth. 2015;15(suppl 2):S7. https:// doi.org/10.1186/1471-2393-15-S2-S7.

10. American Academy of Pediatrics Committee on Fetus and Newborn. Levels of neonatal care. Pediatrics. 2012;130(3):587-97. https://doi.org/10.1542/ peds.2012-1999.

11. World Health Organization (WHO); March of Dimes; The Partnership for Maternal, Newborn \& Child Health; Save the Children. Born too soon: the global action report on preterm birth. Geneva: WHO; 2012. https://apps. who.int/iris/bitstream/handle/10665/44864/9789241503433_eng.pdf; jsessionid=0CCE0D6ECDB78F309AD7A4A14A9CEF0E? sequence $=1$. Accessed 25 Mar 2019 
12. Allen MC, Cristofalo EA, Kim C. Outcomes of preterm infants: morbidity replaces mortality. Clin Perinatol. 2011;38(3):441-54. https://doi.org/10.1016/j. clp.2011.06.011.

13. Ellsbury DL, Clark RH, Ursprung R, Handler DL, Dodd ED, Spitzer AR. A multifaceted approach to improving outcomes in the NICU: the Pediatrix 100000 Babies Campaign. Pediatrics. 2016;137(4):e20150389. https://doi.org/ 10.1542/peds.2015-0389.

14. O'Brien BC, Harris IB, Beckman TJ, Reed DA, Cook DA. Standards for reporting qualitative research: a synthesis of recommendations. Acad Med. 2014:89(9):1245-51. https://doi.org/10.1097/ACM.0000000000000388.

15. World Health Organization (WHO). Everybody's business: strengthening health systems to improve health outcomes: WHO's framework for action. Geneva: WHO; 2007. http://www.who.int/healthsystems/strategy/ everybodys_business.pdf. Accessed 25 Mar 2019

16. Population Division, Department of Economic and Social Affairs, United Nations (UN). World population prospects: the 2017 revision. In: Key findings and advance tables. New York, NY: UN; 2017. https:/esa.un.org/unpd/wpp/ publications/files/wpp2017_keyfindings.pdf. Accessed 25 Mar 2019.

17. Ministry of Health $(\mathrm{MOH})$, Federal Democratic Republic of Ethiopia. Health Sector Development Programme IV: 2010/11-2014/15. Addis Ababa, Ethiopia: MOH; 2010. http://tucghe.org/HSDP\%20IV.pdf. Accessed 25 Mar 2019

18. Morse JM. The significance of saturation. Qual Health Res. 1995;5(2):147-9. https://doi.org/10.1177/104973239500500201.

19. NVivo qualitative data analysis software [computer program]. Version 12; QSR International Pty Ltd. 2018

20. Central Statistical Agency (CSA), Federal Democratic Republic of Ethiopia; ICF. Ethiopia Demographic and Health Survey 2016. In: Addis Ababa. Ethiopia: CSA and ICF; 2016. https://dhsprogram.com/pubs/pdf/ FR328/FR328.pdf.

21. Population and Housing Census of Ethiopia Administrative Report. Addis Ababa, Ethiopia: Central Statistical Authority; 2012. http://unstats.un.org/ unsd/censuskb20/Attachment489.aspx?AttachmentType

22. Manyazewal T, Oosthuizen MJ, Matlakala MC. Proposing evidence-based strategies to strengthen implementation of healthcare reform in resourcelimited settings: a summative analysis. BMJ Open. 2016;6(9):e012582. https:// doi.org/10.1136/bmjopen-2016-012582.

23. Olukoga A, Bachmann M, Harris G, Olukoga T, Oluwadiya K. Analysis of the perception of institutional function for health sector reform in Nigeria. Int Health. 2010;2(2):150-5. https://doi.org/10.1016/j.inhe.2009.12.006.

24. Ganjian S, Dowling PT, Hove J, Moreno G. What physicians from diverse specialties know and support in health care reform. Fam Med. 2015;47(4): 283-91 https://www.ncbi.nlm.nih.gov/pmc/articles/PMC4392847/.

25. Ethiopian Public Health Institute (EPHI); Federal Ministry of Health, Federal Democratic Republic of Ethiopia; ICF International. Ethiopia service provision assessment plus survey 2014. Addis Ababa, Ethiopia: EPHI; 2014. https:// www.ephi.gov.et/images/pictures/FINAL\%20draft\%20SPA+\%20\%2 OREPORT\%20survey\%20tools\%20adjusted\%20-\%20Dec\%2024\%20\%202015. pdf. Accessed 25 March 2019

26. Federal Ministry of Health (FMOH), Federal Democratic Republic of Ethiopia. National human resource for health strategic plan for Ethiopia 2016-2025. Addis Ababa, Ethiopia: FMOH; 2016

27. Federal Ministry of Health (FMOH) Federal Democratic Republic of Ethiopia. Basic emergency obstetric and newborn care training manual. Addis Ababa, Ethiopia: MOH; 2018.

28. Ethiopian Public Health Institute (EPHI). Federal Ministry of Health, Federal Democratic Republic of Ethiopia; Averting Maternal Death and Disability, Columbia University Mailman School of Public Health. In: Ethiopian emergency obstetric and newborn care (EmONC) assessment 2016: final report. Addis Ababa, Ethiopia: EPHI; 2017. https://www.ephi.gov.et/images/ pictures/download2010/FINAL-EmONC-Final-Report-Oct25-2017.pdf. Accessed 25 Mar 2019.

29. Federal Ministry of Health (FMOH). Health Sector Transformation Plan. Addis Ababa, Ethiopia: FMOH; 2015

30. Federal Ministry of Health (FMOH). National Health Care Financing Strategy, 2015-2035. Addis Ababa. Ethiopia: FMOH; 2015.

31. Manyazewal T. Using the World Health Organization health system building blocks through survey of healthcare professionals to determine the performance of public healthcare facilities. Arch Public Health. 2017;75:50. https://doi.org/10.1186/s13690-017-0221-9.
32. World Health Organization (WHO). Standards for improving quality of maternal and newborn care in health facilities. Geneva: WHO; 2016. https:// apps.who.int/iris/bitstream/handle/10665/249155/9789241511216-eng.pdf; jsessionid=C8815D1699A9ECB2F08C5AC2B5513F5F? sequence $=1$.

33. Campbell-Yeo M, Deorari A, McMillan DD, et al. Educational barriers of nurses caring for sick and at-risk infants in India. Int Nurs Rev. 2014;61(3): 398-405. https://doi.org/10.1111/inr.12121.

34. Neogi SB, Khanna R, Chauhan M, et al. Inpatient care of small and sick newborns in healthcare facilities. J Perinatol. 2016;36(suppl 3):S18-23. https://doi.org/10.1038/jp.2016.186

35. Brantuo MN, Cristofalo E, Meheš MM et al. Evidence-based training and mentorship combined with enhanced outcomes surveillance to address the leading causes of neonatal mortality at the district hospital level in Ghana. Tropical Med Int Health. 2014;19(4):417-26. https://doi.org/10. 1111/tmi.12270.

36. Hjemås G, Bråthen R, Vikan ST, Haugen JA. Improving quality on health data: recommendations and guidelines. Based on the case of the health management information system in Malawi and DHIS2. Oslo, Norway: Statistisk sentralbyrå (Statistics Norway); 2017. https://www.ssb.no/helse/ artikler-og-publikasjoner/_attachment/291375?_ts=159a77546b0.

37. Ethiopian Public Health Institute (EPHI). National technical guidance for maternal and perinatal death surveillance and response. Addis Ababa, Ethiopia: EPHI; 2017. https://www.ephi.gov.et/images/pictures/NationalMaternal-and-Perinatal\%2D\%2DDeath-Surveillance-and-Responseguidance-2017.pdf.

38. Teklegiorgis K, Tadesse K, Mirutse G, Terefe W. Level of data quality from Health Management Information Systems in a resources limited setting and its associated factors, eastern Ethiopia. S Afr J Inf Manag. 2016;18(1):a612. https://doi.org/10.4102/sajim.v18i1.612.

39. Yarinbab TE, Assefa MK. Utilization of HMIS data and its determinants at health facilities in east Wollega zone, Oromia regional state, Ethiopia: a health facility based cross-sectional study. Res Rev J Med Health Sci. 2018; 7(1):4-9 http://www.rroij.com/open-access/utilization-of-hmis-data-and-itsdeterminants-at-health-facilities-in-east-wollega-zone-oromia-regional-stateethiopia-a-health-fa.pdf.

40. Lydon M, Longwe M, Likomwa D, et al. Starting the conversation: community perspectives on preterm birth and kangaroo mother care in southern Malawi. J Glob Health. 2018;8(1):010703. https://doi.org/10.7189/ jogh.08.010703.

\section{Publisher's Note}

Springer Nature remains neutral with regard to jurisdictional claims in published maps and institutional affiliations.

Ready to submit your research? Choose BMC and benefit from:

- fast, convenient online submission

- thorough peer review by experienced researchers in your field

- rapid publication on acceptance

- support for research data, including large and complex data types

- gold Open Access which fosters wider collaboration and increased citations

- maximum visibility for your research: over $100 \mathrm{M}$ website views per year

At $\mathrm{BMC}$, research is always in progress.

Learn more biomedcentral.com/submission 